\title{
THREE-DIMENSIONAL STEADY-STATE INDENTATION PROBLEM FOR A GENERAL VISCOELASTIC MATERIAL
}

\author{
BY \\ J. M. GOLDEN (Environmental Research Unit, St. Martin's House, Dublin, Ireland) \\ G. A. C. GRAHAM (Simon Fraser University, Burnaby, B. C., Canada) \\ AND \\ Q. LAN (Simon Fraser University, Burnaby, B. C., Canada)
}

\begin{abstract}
The steady-state problem is considered for periodic normal loading by a smooth, rigid indentor on a half-space exhibiting general viscoelastic behaviour. A technique is developed for summing the infinite series that arise. The method is applicable to the case in which the viscoelastic behaviour is described by a discrete spectrum model, in other words, by a finite sum of decaying exponentials. Numerical results are presented for two decay times. The extension to any number of decay times is straightforward.
\end{abstract}

1. Introduction. Recently, Golden and Graham [1] gave the steady-state solution to the problem of a rigid indentor, subject to normal periodic loading, on a viscoelastic half-space. Detailed results were given for the case where the half-space was assumed to respond as a standard linear solid. In that paper, only the plane strain problem was considered. The corresponding three-dimensional problem was discussed subsequently in $[2,3]$. In these papers, it was shown that the contact pressure and indentation obey integral equations where the kernels are infinite sums of terms involving integrals of the viscoelastic functions. For a standard linear solid, the summations can be carried out without difficulty to give closed formulas. In this case, considerable analytical progress can be made before resorting to numerical calculation.

For more general materials, the infinite summations cannot be carried out in an elementary manner. However, in [4], this question was addressed in the context of a different problem, namely that of a fixed length crack in an infinite body under sinusoidal loading. In that paper, the authors showed that the kernels obey certain integral equations, whose solutions can be determined in closed form, at least for discrete spectrum models. The solution of these equations amounts to summing the infinite series. In the present paper, this method is extended to the contact problem.

Received March 3, 1992.

1991 Mathematics Subject Classification. Primary 73F15.

(C)1994 Brown University 
2. General formulation. We consider the following Hertz problem: a rigid indentor with axisymmetric form $S(r)$, is pressed into a lubricated viscoelastic half-space, occupying the region $z>0$, by a time-dependent load $W(t)$. Here $r$ and $z$ are the usual cylindrical coordinates. Because of the axisymmetry of the problem, we can assume that the contact area $C(t)$ is a circle. Let $a(t)$ be the radius of this circle and $u(r, t)$ the normal surface displacement at position $\vec{r}$ and time $t$, and $p(r, t)$ the normal pressure on the surface at $\vec{r}$ and $t$. Then we have (see [5], for example)

$$
v(r, t)=\frac{1}{2 \pi} \int_{C(t)} d s^{\prime} \frac{p\left(r^{\prime}, t\right)}{\left|\vec{r}^{\prime}-\vec{r}\right|}, \quad r \leq a(t),
$$

where

$$
v(r, t)=\int_{-\infty}^{t} d t^{\prime} l\left(t-t^{\prime}\right) u\left(r, t^{\prime}\right)
$$

The inverse of $(2.2)$ is

$$
u(r, t)=\int_{-\infty}^{t} d t^{\prime} k\left(t-t^{\prime}\right) v\left(r, t^{\prime}\right)
$$

Here $d s^{\prime}$ is the surface element at $\vec{r}$ and $l(t)$ and $k(t)$ are singular functions closely related to the relaxation function and creep function of the medium, respectively. They are inverse functions in the sense that

$$
\int_{t_{1}}^{t_{2}} d t^{\prime} l\left(t_{2}-t^{\prime}\right) k\left(t^{\prime}-t_{1}\right)=\int_{t_{1}}^{t_{2}} d t^{\prime} k\left(t_{2}-t^{\prime}\right) l\left(t^{\prime}-t_{1}\right)=\delta\left(t_{2}-t_{1}\right) .
$$

In this paper, we will only consider discrete spectrum models [5]

$$
\begin{aligned}
& l(t)=l_{0} \delta(t)+\sum_{i=1}^{N} l_{i} e^{-\alpha_{i} t}, \\
& k(t)=k_{0} \delta(t)+\sum_{i=1}^{N} k_{i} e^{-\beta_{i} t} .
\end{aligned}
$$

In order to satisfy $(2.4)$, the coefficients $l_{i}, k_{i}, i=1,2, \ldots, N$ must be related by

$$
\begin{gathered}
l_{0} k_{0}=1 ; \\
l_{0}+\sum_{i=1}^{N} \frac{l_{i}}{\alpha_{i}-\beta_{j}}=0, \quad j=1,2, \ldots, N ; \\
k_{0}-\sum_{i=1}^{N} \frac{k_{i}}{\alpha_{j}-\beta_{i}}=0, \quad j=1,2, \ldots, N ; \\
l_{i}=-\left\{\sum_{j=1}^{N} \frac{k_{j}}{\left(\alpha_{i}-\beta_{j}\right)^{2}}\right\}^{-1}, \quad i=1,2, \ldots, N ; \\
k_{i}=-\left\{\sum_{j=1}^{N} \frac{l_{j}}{\left(\alpha_{j}-\beta_{i}\right)^{2}}\right\}^{-1}, \quad i=1,2, \ldots, N .
\end{gathered}
$$


Let $D(t)$ be the indentation at the tip of the indentor into the half-space. Then the surface displacement $u(r, t)$ takes the form

$$
u(r, t)=D(t)-S(r), \quad r \leq a(t) .
$$

We are interested in the case in which the applied load is oscillating in magnitude, so that the contact area radius $a(t)$ will pass through a series of maxima and minima before the current time $t$. Let $S_{G}(t)$ be the set of all these times $t^{\prime} \leq t$ such that $C\left(t^{\prime}\right) \supseteq C(t)$, while $S_{L}(t)$ is its complement in $(-\infty, t]$ and let $\theta_{i}(t), i=$ $1,2,3, \ldots$, denote the times such that $t>\theta_{1}(t)>\theta_{2}(t)>\theta_{3}(t)>\cdots$ and

$$
a\left(\theta_{i}(t)\right)=a(t) .
$$

First we consider the case in which the contact area is shrinking at current time $t$. The hereditary integral $v(r, t)$ can be decomposed, as described in [1,5], to give

$$
v(r, t)=\int_{S_{G}(t)} d t^{\prime} \Pi_{G}\left(t, t^{\prime}\right) u\left(r, t^{\prime}\right)+\int_{S_{L}(t)} d t^{\prime} \Pi_{L}\left(t, t^{\prime}\right) v\left(r, t^{\prime}\right),
$$

where

$$
\begin{aligned}
& \Pi_{G}\left(t, t^{\prime}\right)=T_{0}\left(t, t^{\prime}\right) R\left(t^{\prime} ; \theta_{1}(t), t\right)+T_{2}\left(t, t^{\prime}\right) R\left(t^{\prime} ; \theta_{3}(t), \theta_{2}(t)\right)+\cdots, \\
& \Pi_{L}\left(t, t^{\prime}\right)=T_{1}\left(t, t^{\prime}\right) R\left(t^{\prime} ; \theta_{2}(t), \theta_{1}(t)\right)+T_{3}\left(t, t^{\prime}\right) R\left(t^{\prime} ; \theta_{4}(t), \theta_{3}(t)\right)+\cdots
\end{aligned}
$$

The functions $T_{i}\left(t, t^{\prime}\right)$ are defined as follows:

$$
\begin{gathered}
T_{0}\left(t, t^{\prime}\right)=l\left(t-t^{\prime}\right), \\
T_{i}\left(t, t^{\prime}\right)=\left\{\begin{array}{lc}
\int_{t^{\prime}}^{\theta_{i}(t)} d t^{\prime \prime} T_{i-1}\left(t, t^{\prime \prime}\right) k\left(t^{\prime \prime}-t^{\prime}\right), & i \text { odd }, \\
\int_{t^{\prime}}^{\theta^{\prime}(t)} d t^{\prime \prime} T_{i-1}\left(t, t^{\prime \prime}\right) l\left(t^{\prime \prime}-t^{\prime}\right), & i \text { even, },
\end{array}\right.
\end{gathered}
$$

while function $R\left(t ; t_{2}, t_{1}\right)$ is given by

$$
R\left(t ; t_{2}, t_{1}\right)= \begin{cases}1, & t \in\left[t_{2}, t_{1}\right] \\ 0, & t \notin\left[t_{2}, t_{1}\right]\end{cases}
$$

for all $t_{2}, t_{1}, t$. According to the definition of $S_{G}(t)$, we know that $r$ is in the contact area for any time $t^{\prime} \in S_{G}(t)$ if it is there at time $t$. Therefore $u\left(r, t^{\prime}\right)$ is known to be $D\left(t^{\prime}\right)-S(r)$ for any time $t^{\prime} \in S_{G}(t)$ and the first term in equation (2.14) is given by

$$
\begin{aligned}
\int_{S_{G}(t)} d t^{\prime} \Pi_{G}\left(t, t^{\prime}\right) u\left(r, t^{\prime}\right) & =\int_{S_{G}(t)} d t^{\prime} \Pi_{G}\left(t, t^{\prime}\right)\left(D\left(t^{\prime}\right)-S(r)\right) \\
& =D_{c}(t)-S(r) \Pi_{G}(t)
\end{aligned}
$$

for $r$ belonging to the contact area at time $t$, where

$$
\begin{aligned}
D_{c}(t) & =\int_{S_{G}(t)} d t^{\prime} \Pi_{G}\left(t, t^{\prime}\right) D\left(t^{\prime}\right), \\
\Pi_{G}(t) & =\int_{S_{G}(t)} d t^{\prime} \Pi_{G}\left(t, t^{\prime}\right) .
\end{aligned}
$$


Furthermore, for time $t^{\prime} \in S_{L}(t)$, the contact region $C\left(t^{\prime}\right)$ is always contained in $C(t)$. Hence we can interchange the time and space integration to put the second integral of equation (2.14) in the form

$$
\int_{S_{L}(t)} d t^{\prime} \Pi_{L}\left(t, t^{\prime}\right) v\left(r, t^{\prime}\right)=\frac{1}{2 \pi} \int_{C(t)} d s^{\prime} \frac{q_{c}\left(r^{\prime}, t\right)}{\left|\vec{r}^{\prime}-\vec{r}\right|}, \quad r \leq a(t)
$$

where

$$
q_{c}(r, t)=\int_{S_{L}(t)} d t^{\prime} \Pi_{L}\left(t, t^{\prime}\right) p\left(r, t^{\prime}\right) .
$$

Therefore equation (2.14) implies that

$$
v(r, t)=v_{c}(r, t)+\frac{1}{2 \pi} \int_{C(t)} d s^{\prime} \frac{q_{c}\left(r^{\prime}, t\right)}{\left|\vec{r}^{\prime}-\vec{r}\right|}, \quad r \leq a(t),
$$

where

$$
v_{c}(r, t)=D_{c}(t)-S(r) \Pi_{G}(t) .
$$

Substituting (2.1) into (2.25) gives us

$$
v_{c}(r, t)=\frac{1}{2 \pi} \int_{C(t)} d s^{\prime} \frac{p\left(r^{\prime}, t\right)-q_{c}\left(r^{\prime}, t\right)}{\left|\vec{r}^{\prime}-\vec{r}\right|}, \quad r \leq a(t) .
$$

If we define a quantity $D_{e}(t)$ such that

$$
D_{c}(t)=\int_{S_{G}(t)} d t^{\prime} \Pi_{G}\left(t, t^{\prime}\right) D\left(t^{\prime}\right)=\Pi_{G}(t) D_{e}(t),
$$

then we have

$$
v_{c}(r, t)=\Pi_{G}(t)\left(D_{e}(t)-S(r)\right),
$$

and equation (2.27) takes the form

$$
\Pi_{G}(t)\left(D_{e}(t)-S(r)\right)=\frac{1}{2 \pi} \int_{C(t)} d s^{\prime} \frac{p\left(r^{\prime}, t\right)-q_{c}\left(r^{\prime}, t\right)}{\left|\vec{r}^{\prime}-\vec{r}\right|}, \quad r \leq a(t) .
$$

This is a standard elastic form. Recalling the elastic solution [5], we get

$$
p(r, t)=q_{c}(r, t)+k_{e} \Pi_{G}(t) p_{e}(r, t)
$$

where $p_{e}(r, t)$ is the pressure distribution on an elastic medium characterized by Lamé constants $\lambda, \mu$ where $k_{e}=\frac{\lambda+2 \mu}{2 \mu(\lambda+\mu)}$. For the viscoelastic problem, $k_{e}$ can be freely chosen but the quantities $k_{e} p_{e}(r, t)$ and $k_{e} W_{e}(t)$ are determined. Here $k_{e}$ is chosen to be the long time modulus

$$
k_{e}=\frac{1}{l_{e}}=\int_{0}^{\infty} k\left(t^{\prime}\right) d t^{\prime}=k_{0}+\sum_{i=1}^{N} \frac{k_{i}}{\beta_{i}}=\left\{l_{0}+\sum_{i=1}^{N} \frac{l_{i}}{\alpha_{i}}\right\}^{-1} .
$$

This choice of $k_{e}$ allows us to make connections with the results of [6] in Secs. 3 and 4. 
For the second case in which $C(t)$ is expanding, we begin with the decomposition of (2.3). After similar manipulations, we obtain

$$
\begin{aligned}
& \int_{S_{L}(t)} d t^{\prime} \Gamma_{L}\left(t, t^{\prime}\right) p\left(r, t^{\prime}\right)=k_{e}\left(1-\Gamma_{G}(t)\right) p_{e}(r, t), \\
& D(t)=\int_{S_{G}(t)} d t^{\prime} \Gamma_{G}\left(t, t^{\prime}\right) D\left(t^{\prime}\right)+D_{e}(t)\left(1-\Gamma_{G}(t)\right),
\end{aligned}
$$

where

$$
\begin{aligned}
& \Gamma_{L}\left(t, t^{\prime}\right)=N_{0}\left(t, t^{\prime}\right) R\left(t^{\prime} ; \theta_{1}(t), t\right)+N_{2}\left(t, t^{\prime}\right) R\left(t^{\prime} ; \theta_{3}(t), \theta_{2}(t)\right)+\cdots, \\
& \Gamma_{G}\left(t, t^{\prime}\right)=N_{1}\left(t, t^{\prime}\right) R\left(t^{\prime} ; \theta_{2}(t), \theta_{1}(t)\right)+N_{3}\left(t, t^{\prime}\right) R\left(t^{\prime} ; \theta_{4}(t), \theta_{3}(t)\right)+\cdots,
\end{aligned}
$$

and

$$
\Gamma_{G}(t)=\int_{S_{G}(t)} d t^{\prime} \Gamma_{G}\left(t, t^{\prime}\right)
$$

Here the functions $N_{i}\left(t, t^{\prime}\right)$ are given by

$$
\begin{gathered}
N_{0}\left(t, t^{\prime}\right)=k\left(t-t^{\prime}\right), \\
N_{i}\left(t, t^{\prime}\right)= \begin{cases}\int_{t^{\prime}}^{\theta_{(}(t)} d t^{\prime \prime} N_{i-1}\left(t, t^{\prime \prime}\right) l\left(t^{\prime \prime}-t^{\prime}\right), & i \text { odd }, \\
\int_{t^{\prime}}^{\theta_{(}(t)} d t^{\prime \prime} N_{i-1}\left(t, t^{\prime \prime}\right) k\left(t^{\prime \prime}-t^{\prime}\right), & i \text { even . }\end{cases}
\end{gathered}
$$

Integrating (2.31) and (2.33) gives us integral equations for the total load

$$
\begin{gathered}
W(t)=\int_{C(t)} d s q_{c}\left(r^{\prime}, t\right)+k_{e} \Pi_{G}(t) W_{e}(t), \quad \text { for } C(t) \text { contracting, } \\
\int_{S_{L}(t)} d t^{\prime} \Gamma_{L}\left(t, t^{\prime}\right) W\left(t^{\prime}\right)=k_{e}\left(1-\Gamma_{G}(t)\right) W_{e}(t), \quad \text { for } C(t) \text { expanding. }
\end{gathered}
$$

Now let us consider the periodic solution of this problem. We choose one period $\left[\Delta_{1}, \Delta_{2}\right]$ such that $\Delta_{2}-\Delta_{1}=\Delta$, which is the period, and where at times $\Delta_{1}, \Delta_{2}$ the contact region $C(t)$ is maximum. Also, we assume that there is only one time, say $t_{0}$, in this period, when $C(t)$ is minimum.

For the contracting phase $t \in\left[\Delta_{1}, t_{0}\right]$, let $t_{1}(t)$ be the solution to equation $a\left(t_{1}(t)\right)=a(t)$ in $\left[t_{0}, \Delta_{2}\right]$. Then we can express $\theta_{i}$ in terms of $t$ and $t_{1}(t)$ as follows:

$$
\theta_{1}(t)=t_{1}(t)-\Delta ; \quad \theta_{2}(t)=t-\Delta ; \quad \theta_{3}(t)=t_{1}(t)-2 \Delta
$$

and so on. It follows that $p(r, t), W(t)$, and $D(t)$ in the decreasing phase $\left[\Delta_{1}, t_{0}\right]$, satisfy the following integral equations:

$$
\begin{gathered}
p(r, t)=\int_{t}^{t_{1}(t)} d t^{\prime} \Pi_{L}^{(p)}\left(t, t^{\prime}\right) p\left(r, t^{\prime}\right)+k_{e} \Pi_{G}^{(p)}(t) p_{e}(r, t), \\
W(t)=\int_{t}^{t_{1}(t)} d t^{\prime} \Pi_{L}^{(p)}\left(t, t^{\prime}\right) W\left(t^{\prime}\right)+k_{e} \Pi_{G}^{(p)}(t) W_{e}(t), \\
\int_{t_{1}(t)-\Delta}^{t} d t^{\prime} \Pi_{G}^{(p)}\left(t, t^{\prime}\right) D\left(t^{\prime}\right)=\Pi_{G}^{(p)}(t) D_{e}(t),
\end{gathered}
$$


where

$$
\begin{aligned}
\Pi_{L}^{(p)}\left(t, t^{\prime}\right) & =\sum_{k=1}^{\infty} T_{2 k-1}\left(t, t^{\prime}-k \Delta\right), \\
\Pi_{G}^{(p)}\left(t, t^{\prime}\right) & =\sum_{k=0}^{\infty} T_{2 k}\left(t, t^{\prime}-k \Delta\right) \\
\Pi_{G}^{(p)}(t) & =\int_{t_{1}(t)-\Delta}^{t} d t^{\prime} \Pi_{G}^{(p)}\left(t, t^{\prime}\right) .
\end{aligned}
$$

For the expanding phase, i.e. $t \in\left[t_{0}, \Delta_{2}\right]$, we get from (2.33), (2.34), and (2.41) that

$$
\begin{gathered}
\int_{t_{1}(t)}^{t} d t^{\prime} \Gamma_{L}^{(p)}\left(t, t^{\prime}\right) p\left(\vec{r}, t^{\prime}\right)=k_{e}\left(1-\Gamma_{G}^{(p)}(t)\right) p_{e}(\vec{r}, t) \\
\int_{t_{1}(t)}^{t} d t^{\prime} \Gamma_{L}^{(p)}\left(t, t^{\prime}\right) W\left(t^{\prime}\right)=k_{e}\left(1-\Gamma_{G}^{(p)}(t)\right) W_{e}(t) \\
D(t)=\int_{t-\Delta}^{t_{1}(t)} d t^{\prime} \Gamma_{G}^{(p)}\left(t, t^{\prime}\right) D\left(t^{\prime}\right)+\left(1-\Gamma_{G}^{(p)}(t)\right) D_{e}(t)
\end{gathered}
$$

where

$$
\begin{aligned}
\Gamma_{L}^{(p)}\left(t, t^{\prime}\right) & =\sum_{k=0}^{\infty} N_{2 k}\left(t, t^{\prime}-k \Delta\right), \\
\Gamma_{G}^{(p)}\left(t, t^{\prime}\right) & =\sum_{k=0}^{\infty} N_{2 k+1}\left(t, t^{\prime}-k \Delta\right), \\
\Gamma_{G}^{(p)}(t) & =\int_{t-\Delta}^{t_{1}(t)} d t^{\prime} \Gamma_{G}^{(p)}\left(t, t^{\prime}\right) .
\end{aligned}
$$

In this case the $\theta_{i}(t)$ are given by

$$
\theta_{1}(t)=t_{1}(t), \quad \theta_{2}(t)=t-\Delta, \quad \theta_{3}(t)=t_{1}(t)-\Delta,
$$

and so on where $t_{1}(t)$ is the solution of $a\left(t_{1}(t)\right)=a(t)$ in $\left[\Delta_{1}, t_{0}\right]$. The steady-state contact problem is thus expressed in terms of the six integral equations (2.43)-(2.45) and (2.49) $-(2.51)$, four of them independent. These equations involve solutions to the corresponding elastic problem indicated by the subscript " $e$ ". For a spherical indentor of large radius $R$,

and the elastic solutions satisfy

$$
S(r)=\frac{r^{2}}{2 R}
$$

$$
\begin{aligned}
p_{e}(r, t) & =\frac{4}{\pi R k_{e}}\left(a^{2}(t)-r^{2}\right)^{1 / 2}, \\
W_{e}(t) & =\frac{8 a^{3}(t)}{3 k_{e} R}, \\
D_{e}(t) & =\frac{a^{2}(t)}{R} .
\end{aligned}
$$


3. Theoretical results. In the last section, the contact problem was reduced to the solution of six integral equations. In the case of a standard linear material, evaluation of the kernels of these integral equations can be reduced to summation of geometric series. Then these six integral equations can be changed into three ordinary differential equations and three algebraic equations. Details of this work may be found in [1,2]. For more general material behaviour that method breaks down. However, a technique, previously used for a crack problem [4], is applicable.

Let us first consider the kernel $\Pi_{L}^{(p)}\left(t, t^{\prime}\right)$ given by (2.46). According to the definition of $T_{n}\left(t, t^{\prime}\right)$, we have

$$
T_{n}\left(t, t^{\prime}\right)=\int_{t^{\prime}}^{\theta_{n}(t)} d t^{\prime \prime \prime} \int_{t^{\prime \prime \prime}}^{\theta_{n-1}(t)} d t^{\prime \prime} T_{n-2}\left(t, t^{\prime \prime}\right) l\left(t^{\prime \prime}-t^{\prime \prime \prime}\right) k\left(t^{\prime \prime \prime}-t^{\prime}\right)
$$

for odd numbers $n \geq 3$. The integral over $t^{\prime \prime}$ can be extended at the lower limit to $t^{\prime}$ since $l\left(t^{\prime \prime}-t^{\prime \prime \prime}\right)$ vanishes over this interval. This allows the order of integrations to be interchanged without difficulty and one has (omitting explicit mention of the $t$ dependence of $\theta_{n}$ )

$$
T_{n}\left(t, t^{\prime}\right)=\int_{t^{\prime}}^{\theta_{n-1}} d t^{\prime \prime} T_{n-2}\left(t, t^{\prime \prime}\right) G_{n}\left(t^{\prime \prime}, t^{\prime}\right)
$$

where

$$
G_{n}\left(t^{\prime \prime}, t^{\prime}\right)=\int_{t^{\prime}}^{\theta_{n}} d t^{\prime \prime \prime} l\left(t^{\prime \prime}-t^{\prime \prime \prime}\right) k\left(t^{\prime \prime \prime}-t^{\prime}\right)
$$

Using the inverse relationship (2.4) between $k(t)$ and $l(t)$, one deduces that

$$
G_{n}\left(t^{\prime \prime}, t^{\prime}\right)=\delta\left(t^{\prime \prime}-t^{\prime}\right), \quad t^{\prime \prime} \leq \theta_{n} \text {. }
$$

Therefore

$$
T_{n}\left(t, t^{\prime}\right)=T_{n-2}\left(t, t^{\prime}\right)+\int_{\theta_{n}}^{\theta_{n-1}} d t^{\prime \prime} T_{n-2}\left(t, t^{\prime \prime}\right) G_{n}\left(t^{\prime \prime}, t^{\prime}\right), \quad t^{\prime} \leq \theta_{n} .
$$

Making the subscript explicitly odd, we can write

$$
T_{2 i-1}\left(t, t^{\prime}-i \Delta\right)=T_{2 i-3}\left(t, t^{\prime}-i \Delta\right)+\int_{t_{1}-i \Delta}^{t-(i-1) \Delta} d t^{\prime \prime} T_{2 i-3}\left(t, t^{\prime \prime}\right) G_{2 i-1}\left(t^{\prime \prime}, t^{\prime}-i \Delta\right),
$$

by using $\theta_{2 n-1}=t_{1}-n \Delta$ and $\theta_{2 n-2}=t-(n-1) \Delta$, where

$$
\begin{aligned}
G_{2 i-1}\left(t^{\prime \prime}, t^{\prime}-i \Delta\right) & =\int_{t^{\prime}-i \Delta}^{t_{1}-i \Delta} d t^{\prime \prime \prime} l\left(t^{\prime \prime}-t^{\prime \prime \prime}\right) k\left(t^{\prime \prime \prime}-t^{\prime}+i \Delta\right) \\
& =\int_{t^{\prime}-\Delta}^{t_{1}-\Delta} d u l\left(t^{\prime \prime}-u+(i-1) \Delta\right) k\left(u-t^{\prime}+\Delta\right) \\
& =G\left(t^{\prime \prime}+(i-1) \Delta, t^{\prime}-\Delta\right) .
\end{aligned}
$$

Here the transformation of variables $u=t^{\prime \prime \prime}+(i-1) \Delta$ is employed. The function

$$
G\left(t^{\prime \prime}, t^{\prime}\right)=\int_{t^{\prime}}^{\theta_{1}} d u l\left(t^{\prime \prime}-u\right) k\left(u-t^{\prime}\right)
$$


has the same functional form as $T_{1}\left(t, t^{\prime}\right)$ with $t^{\prime \prime}$ replacing $t$ but $\theta_{1}(t)$ left untouched. Using (3.6) and (3.7), we have

$$
T_{2 i-1}\left(t, t^{\prime}-i \Delta\right)=T_{2 i-3}\left(t, t^{\prime}-i \Delta\right)+\int_{t_{1}-\Delta}^{t} d u T_{2 i-3}(t, u-(i-1) \Delta) G\left(u, t^{\prime}-\Delta\right) .
$$

Therefore $\Pi_{L}^{(p)}\left(t, t^{\prime}\right)$, given by (2.46), obeys the equation

$$
\Pi_{L}^{(p)}\left(t, t^{\prime}\right)=T_{1}\left(t, t^{\prime}-\Delta\right)+\Pi_{L}^{(p)}\left(t, t^{\prime}-\Delta\right)+\int_{t_{1}-\Delta}^{t} d u \Pi_{L}^{(p)}(t, u) G\left(u, t^{\prime}-\Delta\right) .
$$

Thus

$$
\Pi_{L}^{(p)}\left(t, t^{\prime}-\Delta\right)=T_{1}\left(t, t^{\prime}-2 \Delta\right)+\Pi_{L}^{(p)}\left(t, t^{\prime}-2 \Delta\right)+\int_{t_{1}-\Delta}^{t} d u \Pi_{L}^{(p)}(t, u) G\left(u, t^{\prime}-2 \Delta\right)
$$

and so on. Repeated substitution of (3.11) and its successors into (3.10) together with the assumption, which will be justified later, that

$$
\lim _{n \rightarrow \infty} \Pi_{L}^{(p)}\left(t, t^{\prime}-n \Delta\right)=0,
$$

finally gives an integral equation for $\Pi_{L}^{(p)}\left(t, t^{\prime}\right)$ of the form

$$
\Pi_{L}^{(p)}\left(t, t^{\prime}\right)=K\left(t, t^{\prime}\right)+\int_{\theta_{1}}^{t} d u \Pi_{L}^{(p)}(t, u) K\left(u, t^{\prime}\right)
$$

where

$$
K\left(u, t^{\prime}\right)=\sum_{n=1}^{\infty} G\left(u, t^{\prime}-n \Delta\right) .
$$

We recall that $G\left(t, t^{\prime}-n \Delta\right)=T_{1}\left(t, t^{\prime}-n \Delta\right)$. Now we can solve this integral equation for kernel $\Pi_{L}^{(p)}\left(t, t^{\prime}\right)$. For discrete spectrum models (2.5) and (2.6), we obtain from (3.8) that

$$
G\left(u, t^{\prime}\right)=\sum_{i, j=1}^{N} \frac{l_{i} k_{j}}{\alpha_{i}-\beta_{j}} e^{-\alpha_{i}\left(u-\theta_{1}\right)-\beta_{j}\left(\theta_{1}-t^{\prime}\right)}, \quad u>\theta_{1}(t) .
$$

Then, equation (3.14) takes the form

$$
K\left(u, t^{\prime}\right)=\sum_{i, j=1}^{N} K_{i j} e^{-\alpha_{i}\left(u-\theta_{1}\right)-\beta_{j}\left(\theta_{1}-t^{\prime}\right)},
$$

where

$$
K_{i j}=\frac{l_{i} k_{j}}{\alpha_{i}-\beta_{j}} \frac{e^{-\beta_{j} \Delta}}{1-e^{-\beta_{j} \Delta}} .
$$

To solve (3.13), we make the ansatz for $\Pi_{L}^{(p)}\left(t, t^{\prime}\right)$ of the form

$$
\Pi_{L}^{(p)}\left(t, t^{\prime}\right)=\sum_{i, j=1}^{N} P_{i j}(t) e^{\beta_{j} t^{\prime}}
$$


which clearly obeys (3.12). Substitution into (3.13) gives

$$
\sum_{i=1}^{N} P_{i j}=\sum_{i=1}^{N} K_{i j} e^{-\alpha_{i}\left(t-\theta_{1}\right)-\beta_{j} \theta_{1}}+\sum_{i, m, n=1}^{N} \frac{P_{i m} K_{n j}}{\beta_{m}-\alpha_{n}}\left\{e^{\beta_{m} t-\alpha_{n}\left(t-\theta_{1}\right)-\beta_{j} \theta_{1}}-e^{\left(\beta_{m}-\beta_{j}\right) \theta_{1}}\right\} .
$$

This algebraic equation will certainly be satisfied if a stronger condition is imposed that cancellation takes place term by term in the variable $i$. This gives us the matrix equation

$$
P=K_{1}+P A K_{2},
$$

where $P$ is a square matrix formed by $P_{i j}$ while

$$
\begin{aligned}
\left(K_{1}\right)_{i j} & =K_{i j} e^{-\alpha_{i}\left(t-\theta_{1}\right)-\beta_{j} \theta_{1}}, \\
\left(K_{2}\right)_{n j} & =K_{n j} e^{-\beta_{j} \theta_{1}}, \\
A_{m n} & =\frac{e^{-\alpha_{n}\left(t-\theta_{1}\right)+\beta_{m} t}-e^{\beta_{m} \theta_{1}}}{\beta_{m}-\alpha_{n}} .
\end{aligned}
$$

The formal solution of $(3.20)$ is

$$
P=K_{1}\left(I-A K_{2}\right)^{-1} \text {. }
$$

Similarly, we get

$$
\Pi_{G}^{(p)}\left(t, t^{\prime}\right)=l_{0} \delta\left(t-t^{\prime}\right)+\sum_{i, j=1}^{N} Q_{i j}(t) e^{\alpha_{t^{\prime}}},
$$

where $Q_{i j}(t)$ are the components of a square matrix $Q$ given by

$$
Q=L_{1}\left(I-B L_{2}\right)^{-1} \text {, }
$$

with

$$
\begin{aligned}
\left(L_{1}\right)_{i j} & =\frac{\delta_{i j} l_{j} e^{-\alpha_{j} t}}{1-e^{-\alpha_{j} \Delta}}, \\
\left(L_{2}\right)_{i j} & =\frac{k_{i} l_{j} e^{-\alpha_{j} \theta_{2}}}{\beta_{i}-\alpha_{j}} \frac{e^{-\alpha_{j} \Delta}}{1-e^{-\alpha_{j} \Delta}}, \\
B_{i j} & =\frac{e^{\left(\alpha_{i}-\beta_{j}\right) \theta_{1}+\beta_{j} \theta_{2}}-e^{\alpha_{i} \theta_{2}}}{\alpha_{i}-\beta_{j}} .
\end{aligned}
$$

By comparing the definition of $\Pi_{G}^{(p)}\left(t, t^{\prime}\right)$ and $\Gamma_{L}^{(p)}\left(t, t^{\prime}\right)$, we find that $\Gamma_{L}^{(p)}\left(t, t^{\prime}\right)$ may be obtained from $\Pi_{G}^{(p)}\left(t, t^{\prime}\right)$ by interchanging the roles of $l(t)$ and $k(t)$. Furthermore we see that $\Gamma_{G}^{(p)}\left(t, t^{\prime}-\Delta\right)$ is the same as $\Pi_{L}^{(p)}\left(t, t^{\prime}\right)$ if the roles of $l(t)$ and $k(t)$ are interchanged. Therefore, for time $t \in\left[t_{0}, \Delta_{2}\right]$, we have

$$
\Gamma_{L}^{(p)}\left(t, t^{\prime}\right)=k_{0} \delta\left(t-t^{\prime}\right)+\sum_{i, j=1}^{N} \widehat{Q}_{i j}(t) e^{\beta_{j} t^{\prime}}
$$


and

$$
\Gamma_{G}^{(p)}\left(t, t^{\prime}\right)=\sum_{i, j=1}^{N} \widehat{P}_{i j}(t) e^{\alpha_{j}\left(t^{\prime}+\Delta\right)}
$$

where $\widehat{P}, \widehat{Q}$ are obtained from (3.24) and (3.26), respectively, by interchanging the roles of $l(t)$ and $k(t)$.

Putting expression (3.25) into (2.48) and (3.31) into (2.54), we get

$$
\Pi_{G}^{(p)}(t)=l_{0}+\sum_{i, j=1}^{N} Q_{i j}(t) \frac{e^{\alpha_{j} t}-e^{\alpha_{j} \theta_{1}}}{\alpha_{j}},
$$

and

$$
\Gamma_{G}^{(p)}(t)=\sum_{i, j=1}^{N} \widehat{P}_{i j}(t) \frac{e^{\alpha_{j}\left(\theta_{1}+\Delta\right)}-e^{\alpha_{j}\left(\theta_{2}+\Delta\right)}}{\alpha_{j}} .
$$

Substituting all the kernels into the periodic solution equations (2.43)-(2.45) and (2.49)-(2.51), we have

$$
\begin{aligned}
p(r, t)= & \sum_{i, j=1}^{N} P_{i j}(t) \int_{t}^{t_{1}(t)} d t^{\prime} e^{\beta_{j} t^{\prime}} p\left(r, t^{\prime}\right) \\
& +k_{e}\left\{l_{0}+\sum_{i, j=1}^{N} Q_{i j}(t) \frac{e^{\alpha_{j} t}-e^{\alpha_{j}\left(t_{1}-\Delta\right)}}{\alpha_{j}}\right\} p_{e}(r, t) \text { for } t \in\left[\Delta_{1}, t_{0}\right], \\
k_{0} p(r, & t)+\sum_{i, j=1}^{N} \widehat{Q}_{i j}(t) \int_{t_{1}(t)}^{t} d t^{\prime} e^{\beta_{j} t^{\prime}} p\left(r, t^{\prime}\right) \\
= & k_{e}\left\{1-\sum_{i, j=1}^{N} \widehat{P}_{i j}(t) \frac{e^{\alpha_{j}\left(\Delta+t_{1}\right)}-e^{\alpha_{j} t}}{\alpha_{j}}\right\} p_{e}(r, t) \text { for } t \in\left[t_{0}, \Delta_{2}\right] \\
W(t)= & \sum_{i, j=1}^{N} P_{i j}(t) \int_{t}^{t_{1}(t)} d t^{\prime} e^{\beta_{j} t^{\prime}} W\left(t^{\prime}\right) \\
& +k_{e}\left\{l_{0}+\sum_{i, j=1}^{N} Q_{i j}(t) \frac{e^{\alpha_{j} t}-e^{\alpha_{j}\left(t_{1}-\Delta\right)}}{\alpha_{j}}\right\} W_{e}(t) \text { for } t \in\left[\Delta_{1}, t_{0}\right] \\
= & k_{e}\left\{1-\sum_{i, j=1}^{N} \widehat{P}_{i j}(t) \frac{e^{\alpha_{j}\left(\Delta+t_{1}\right)}-e^{\alpha_{j} t}}{\alpha_{j}}\right\} W_{e}(t) \text { for } t \in\left[t_{0}, \Delta_{2}\right] \\
k_{0} W(t)+ & \sum_{i, j=1}^{N} \widehat{Q}_{i j}(t) \int_{t_{1}(t)}^{t} d t^{\prime} e^{\beta_{j} t^{\prime}} W\left(t^{\prime}\right) \\
& \{
\end{aligned}
$$




$$
\begin{aligned}
l_{0} D(t) & +\sum_{i, j=1}^{N} Q_{i j}(t) \int_{t_{1}-\Delta}^{t} d t^{\prime} e^{\alpha_{j} t^{\prime}} D\left(t^{\prime}\right) \\
= & \left\{l_{0}+\sum_{i, j=1}^{N} Q_{i j}(t) \frac{e^{\alpha_{j} t}-e^{\alpha_{j}\left(t_{1}-\Delta\right)}}{\alpha_{j}}\right\} D_{e}(t) \text { for } t \in\left[\Delta_{1}, t_{0}\right], \\
D(t)= & \sum_{i, j=1}^{N} \widehat{P}_{i j}(t) e^{\alpha_{j} \Delta} \int_{t-\Delta}^{t_{1}} d t^{\prime} e^{\alpha_{j} t^{\prime}} D\left(t^{\prime}\right) \\
& +\left\{1-\sum_{i, j=1}^{N} \widehat{P}_{i j}(t) \frac{e^{\alpha_{j}\left(t_{1}+\Delta\right)}-e^{\alpha_{j} t}}{\alpha_{j}}\right\} D_{e}(t) \text { for } t \in\left[t_{0}, \Delta_{2}\right] .
\end{aligned}
$$

We will now show that $p(r, t), W(t), D(t)$ satisfying the above equations are continuous at time $t_{0}$, and equal at $\Delta_{1}$ and $\Delta_{2}$.

Putting $t=t_{0}$ in (3.36), we have

$$
W\left(t_{0}\right)=k_{e}\left\{l_{0}+\sum_{i, j=1}^{N} Q_{i j}\left(t_{0}\right) \frac{e^{\alpha_{j} t_{0}}-e^{\alpha_{j}\left(t_{0}-\Delta\right)}}{\alpha_{j}}\right\} W_{e}\left(t_{0}\right) .
$$

From (3.29) we know that $B_{i j}\left(t_{0}\right)=0$, since $\theta_{1}=\theta_{2}=t_{0}-\Delta$ for $t=t_{0}$. Therefore it follows from (3.26) that

$$
Q_{i j}\left(t_{0}\right)=\left\{L_{1}\left(t_{0}\right)\right\}_{i j}=\frac{\delta_{i j} l_{j} e^{-\alpha_{j} t_{0}}}{1-e^{-\alpha_{j} \Delta}}
$$

so that

$$
W\left(t_{0}\right)=k_{e}\left(l_{0}+\sum_{i=1}^{N} l_{i} / \alpha_{i}\right) W_{e}\left(t_{0}\right)=W_{e}\left(t_{0}\right)
$$

after using equation (2.32). This agrees with the results given in [6]. Equation (3.37) at $t=t_{0}$ gives us the same result by noting the fact that

$$
\widehat{P}_{i j}\left(t_{0}\right)=\frac{k_{i} l_{j}}{\beta_{i}-\alpha_{j}} \frac{e^{-\alpha_{j}\left(\Delta+t_{0}\right)}}{1-e^{-\alpha_{j} \Delta}}
$$

and using relation (2.9). Therefore $W(t)$ is continuous at time $t_{0}$. Equation (3.36) at $t=\Delta_{1}$ and equation (3.37) at $t=\Delta_{2}$ take the form

$$
\begin{aligned}
& W\left(\Delta_{1}\right)=\sum_{i, j=1}^{N} P_{i j}\left(\Delta_{1}\right) \int_{\Delta_{1}}^{\Delta_{2}} d t^{\prime} e^{\beta_{j} t^{\prime}} W\left(t^{\prime}\right)+k_{e} l_{0} W_{e}\left(\Delta_{1}\right), \\
& W\left(\Delta_{2}\right)=-\frac{1}{k_{0}} \sum_{i, j=1}^{N} \hat{Q}_{i j}\left(\Delta_{2}\right) \int_{\Delta_{1}}^{\Delta_{2}} d t^{\prime} e^{\beta_{j} t^{\prime}} W\left(t^{\prime}\right)+k_{e} l_{0} W_{e}\left(\Delta_{2}\right) .
\end{aligned}
$$

From equation (2.42), we know

$$
t_{1}(t)=\Delta_{2}, \quad \theta_{1}(t)=\Delta_{1}
$$


for time $t=\Delta_{1}$. Therefore, from (3.23), one deduces

$$
A_{i j}\left(\Delta_{1}\right)=0 \text {. }
$$

Thus we can write (3.24) as

$$
P_{i j}\left(\Delta_{1}\right)=\left(K_{1}\right)_{i j}\left(\Delta_{1}\right)=\frac{l_{i} k_{j}}{\alpha_{i}-\beta_{j}} \frac{e^{-\beta_{j} \Delta_{2}}}{1-e^{-\beta_{j} \Delta}} .
$$

Similarly, we obtain

$$
\widehat{Q}_{i j}\left(\Delta_{2}\right)=\frac{\delta_{i j} k_{j} e^{-\beta_{j} \Delta_{2}}}{1-e^{-\beta_{j} \Delta}}
$$

using the fact that $\theta_{1}(t)=\theta_{2}(t)=t_{1}(t)=\Delta_{1}$ and $\widehat{B}_{i j}=0$ for $t=\Delta_{2}$. Therefore

$$
\sum_{i=1}^{N} P_{i j}\left(\Delta_{1}\right)=-\frac{1}{k_{0}} \sum_{i=1}^{N} \widehat{Q}_{i j}\left(\Delta_{2}\right),
$$

by virtue of equation (2.8). Noting $W_{e}\left(\Delta_{1}\right)=W_{e}\left(\Delta_{2}\right)$, we have, from equations (3.44) and (3.45), that $W\left(\Delta_{1}\right)=W\left(\Delta_{2}\right)$. This completes the verification of the periodicity of $W(t)$.

Now we consider $D(t)$. It is clear that $D\left(\Delta_{1}\right)=D\left(\Delta_{2}\right)$ because (3.38) at $t=\Delta_{1}$ gives

$$
D\left(\Delta_{1}\right)=D_{e}\left(\Delta_{1}\right)
$$

equation (3.39) at $t=\Delta_{2}$ yields

$$
D\left(\Delta_{2}\right)=D_{e}\left(\Delta_{2}\right),
$$

and we know that $D_{e}\left(\Delta_{1}\right)=D_{e}\left(\Delta_{2}\right)$. Equations (3.51), (3.52) are in agreement with results given in [6] in a more general context. When $t=t_{0}$, we have, from equations (3.38) and (3.39)

$$
\begin{aligned}
D\left(t_{0}\right)= & -k_{0} \sum_{i=1}^{N} Q_{i j}\left(t_{0}\right) \int_{t_{0}-\Delta}^{t_{0}} d t^{\prime} e^{\alpha_{j} t^{\prime}} D\left(t^{\prime}\right) \\
& +k_{0}\left\{l_{0}+\sum_{i=1}^{N} Q_{i j}\left(t_{0}\right) \frac{e^{\alpha_{j} t_{0}}-e^{\alpha_{j}\left(t_{0}-\Delta\right)}}{\alpha_{j}}\right\} D_{e}\left(t_{0}\right), \\
D\left(t_{0}\right)= & \sum_{i, j=1}^{N} \widehat{P}_{i j}\left(t_{0}\right) e^{\alpha_{j} \Delta} \int_{t_{0}-\Delta}^{t_{0}} d t^{\prime} e^{\alpha_{j} t^{\prime}} D\left(t^{\prime}\right) \\
& +\left\{1-\sum_{i, j=1}^{N} \widehat{P}_{i j}\left(t_{0}\right) e^{\alpha_{j} \Delta} \frac{e^{\alpha_{j} t_{0}}-e^{\alpha_{j}\left(t_{0}-\Delta\right)}}{\alpha_{j}}\right\} D_{e}\left(t_{0}\right) .
\end{aligned}
$$

To prove $D(t)$ is continuous at $t=t_{0}$, it is sufficient to show that

$$
-k_{0} \sum_{i=1}^{N} Q_{i j}\left(t_{0}\right)=\sum_{i=1}^{N} \widehat{P}_{i j}\left(t_{0}\right) e^{\alpha_{j} \Delta} .
$$


This is an immediate consequence of (3.41) and (3.43) with the aid of equation (2.9). The verification of the continuity and periodicity of $p(r, t)$ is the same as that of $W(t)$.

Also, one can show that kernels for the case $N=1$ are the same as the ones obtained in $[1,2]$ by a direct summation.

4. Numerical results. In this section, numerical solutions to integral equations (3.34)-(3.39) by the quadrature method are given for the following three cases:

(i) stress-controlled mode (where the total normal load is specified);

(ii) strain-controlled mode (where the indentation is specified);

(iii) area-controlled mode (where the area of contact is specified).

Here we consider the spherical indentor of large radius $R$, for which the elastic solutions are given by (2.57)-(2.59). All the numerical calculations are carried out for the case $N=2$. The dimensionless quantities $c a(t), c D(t), c^{2} k_{0} W(t), k_{0} p(r, t)$ and the dimensionless parameters $t^{\prime}=\omega t, \beta_{i}^{\prime}=\beta_{i} / \omega, k_{i}^{\prime}=k_{i} /\left(\omega k_{0}\right), \alpha_{i}^{\prime}=\alpha_{i} / \omega$, $l_{i}^{\prime}=l_{i} k_{0} / \omega, i=1,2$, and $k=k_{e} / k_{0}$ are used, where $c=1 /(2 R)$. This method can be applied to the cases when $N>2$ without any difficulty.

(i) Stress-controlled mode. The applied load is assumed to have the simple sinusoidal form

$$
W(t)=K(d-\cos (\omega t)), \quad d \geq 1, \Delta=\frac{2 \pi}{\omega} .
$$

To solve for $a(t), p(r, t), D(t)$, we need to know $t_{0}$ and $\Delta_{1}, \Delta_{2}$. According to [6], $t_{0}=0$ and $\Delta_{2}$, given by (4.30) in [6], now becomes

$$
\tan \left(\omega \Delta_{2}\right)=\left\{\sum_{i=1}^{N} \frac{k_{i} \omega}{\omega^{2}+\beta_{i}^{2}}\right\} /\left\{k_{0}+\sum_{i=1}^{N} \frac{k_{i} \beta_{i}}{\omega^{2}+\beta_{i}^{2}}\right\}
$$

in terms of our parameters here.

Once $t_{0}$ and $\Delta_{1}, \Delta_{2}$ are known, Newton's iteration method is employed to solve the functional equation, obtained by eliminating $W_{e}(t)=W_{e}\left(t_{1}(t)\right)$ from (3.36) and (3.37), for $t_{1}(t)$. Then $W_{e}(t)$ can be obtained from one of these equations. If $W_{e}(t)$ is known, the contact radius $a(t)$, elastic indentation $D_{e}(t)$ and elastic pressure $p_{e}(r, t)$ can be found by using the relations (2.57) to (2.59). Therefore we can get $D(t)$ and $p(r, t)$. For example, the indentation $D(t)$ is obtained by solving equation (3.38) starting at $t=\Delta_{1}$ and equation (3.39) beginning with $t=\Delta_{2}$, using the marching method. Considering that all the kernels are smooth functions, we use the trapezoidal rule for the numerical computations. Results for the stress-controlled mode are presented in Figures 1-3 (see pp. 462-464). From Figures 1 and 2, we can see that $c a(t)$ and $c D(t)$ increase with the value of $k$ and there is very little variation with $\beta_{i}$ and $k_{i}$ if $k$ is close to 1 . Note that, from Figure 1, the minimum contact area occurs at $t=0$ and is only dependent on the value of $k$ and independent of the individual values of $\alpha_{i}^{\prime}$ and $l_{i}^{\prime}$ or $\beta_{i}^{\prime}$ and $k_{i}^{\prime}$. The latter observation follows from (3.42). Figure 2 also shows that the indentation achieves a minimum a little later than the load does for the stress-controlled mode. Contact area and indentation reach their maximum simultaneously and after the load [6]. 


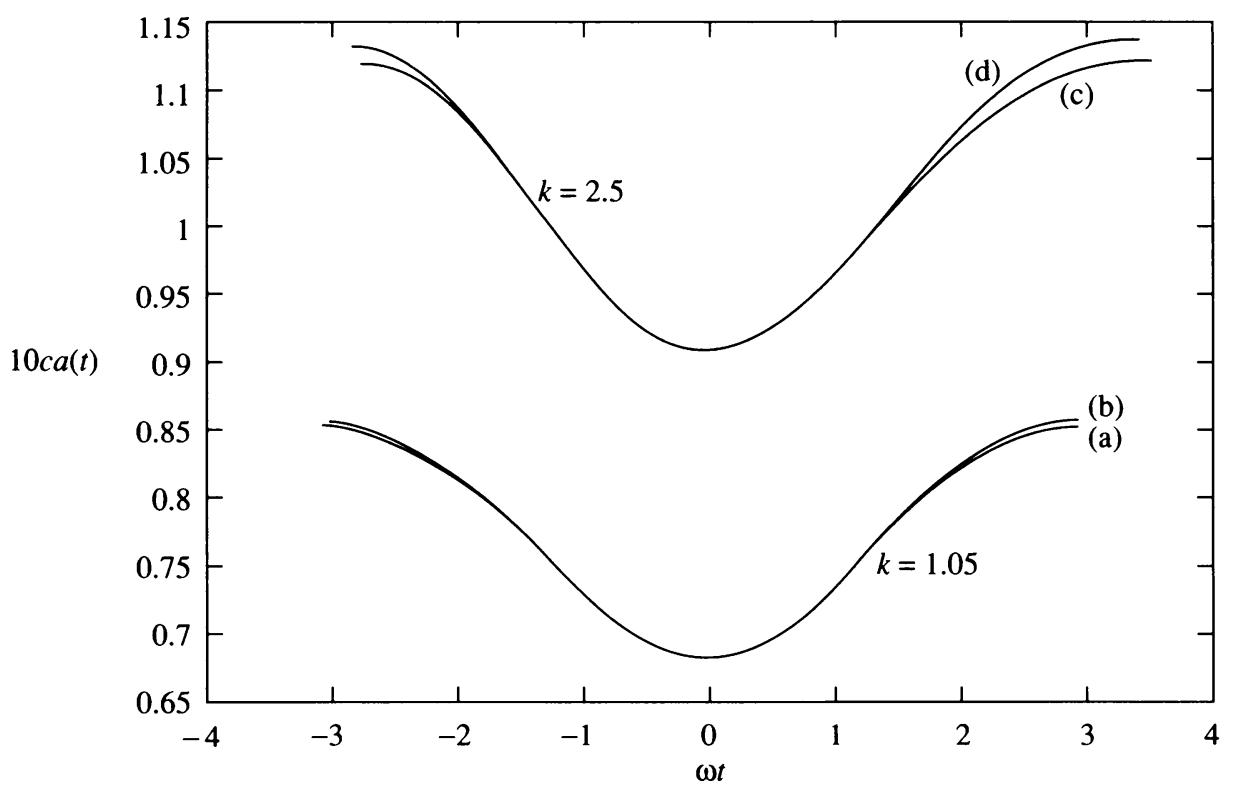

Fig. 1. Contact area radius for the stress-controlled mode. This gives the dimensionless contact area radius $c a(t)$ over a complete cycle under stress-controlled condition for cases (a): $k_{1}^{\prime}=0.0096$, $k_{2}^{\prime}=0.003, \beta_{1}^{\prime}=0.2, \beta_{2}^{\prime}=1.5, k=1.05 ;(\mathrm{b}): k_{1}^{\prime}=0.09$, $k_{2}^{\prime}=0.03, \beta_{1}^{\prime}=2, \beta_{2}^{\prime}=6, k=1.05$; (c): $k_{1}^{\prime}=1.26, k_{2}^{\prime}=0.12$, $\beta_{1}^{\prime}=0.9, \beta_{2}^{\prime}=1.2, k=2.5$; and $(\mathrm{d}): k_{1}^{\prime}=0.52, k_{2}^{\prime}=0.12$, $\beta_{1}^{\prime}=0.4, \beta_{2}^{\prime}=0.6, k=2.5$. Also $c^{2} K k_{0}=0.0008, d=3$.

(ii) Strain-controlled mode. For this case, the indentation is given by

$$
D(t)=N(b-\cos (\omega t)), \quad \Delta=\frac{2 \pi}{\omega} .
$$

We know that the load cannot be negative for the contact problem. To satisfy this condition, the constant $b$ has to be greater or equal to another constant, say $g$, which is given by (4.23) in [6]. For the discrete spectrum model (2.5)

$$
g=k_{e} \sqrt{\left\{\sum_{i=1}^{N} \frac{l_{i} \omega}{\omega^{2}+\alpha_{i}^{2}}\right\}^{2}+\left\{l_{0}+\sum_{i=1}^{N} \frac{l_{i} \alpha_{i}}{\omega^{2}+\alpha_{i}^{2}}\right\}^{2}} .
$$

According to [6], the maximum values of $D_{e}(t)$ and of $a(t)$ occur at the same time as that of $D(t)$. Therefore

$$
\Delta_{2}=-\Delta_{1}=\frac{\pi}{\omega} .
$$

By virtue of (4.25) of [6], the quantity $t_{0}$ satisfies

$$
\tan \left(\omega t_{0}\right)=\left\{\sum_{i=1}^{N} \frac{l_{i} \omega}{\omega^{2}+\alpha_{i}^{2}}\right\} /\left\{l_{0}+\sum_{i=1}^{N} \frac{l_{i} \alpha_{i}}{\omega^{2}+\alpha_{i}^{2}}\right\},
$$

in terms of our present notation. To get $t_{1}(t)$, we eliminate $D_{e}(t)=D_{e}\left(t_{1}(t)\right)$ from equations (3.38) and (3.39) to give a functional equation for $t_{1}(t)$. Then one can 


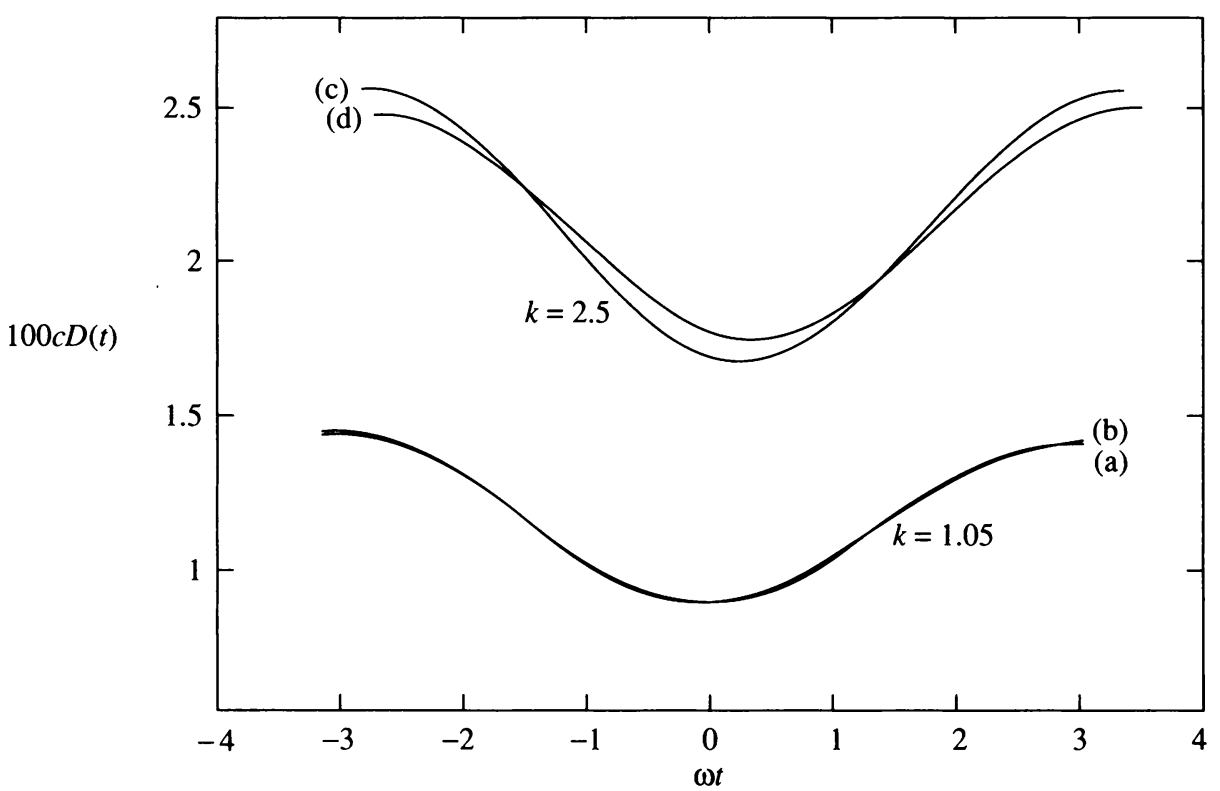

Fig. 2. Indentation for the stress-controlled mode. This gives the dimensionless indentation $c D(t)$ over a complete cycle under stresscontrolled condition for the same cases as those in Fig. 1 (a): $k_{1}^{\prime}=$ $0.0096, k_{2}^{\prime}=0.003, \beta_{1}^{\prime}=0.2, \beta_{2}^{\prime}=1.5, k=1.05 ;(\mathrm{b}): k_{1}^{\prime}=$ $0.09, k_{2}^{\prime}=0.03, \beta_{1}^{\prime}=2, \beta_{2}^{\prime}=6, k=1.05 ;(\mathrm{c}): k_{1}^{\prime}=1.26$, $k_{2}^{\prime}=0.12, \beta_{1}^{\prime}=0.9, \beta_{2}^{\prime}=1.2, k=2.5$; and (d): $k_{1}^{\prime}=0.52$, $k_{2}^{\prime}=0.12, \beta_{1}^{\prime}=0.4, \beta_{2}^{\prime}=0.6, k=2.5$. Also $c^{2} K k_{0}=0.0008$, $d=3$.

find $t_{1}(t)$ by solving this functional equation using an iteration method. If $t_{1}(t)$ is known, $D_{e}(t)$ can be determined by either (3.38) or (3.39). Then $a(t), W(t)$, and $p(r, t)$ can be obtained by the same procedure as before. Numerical results for this case are presented in Figures 4-6 (see pp. 465, 466).

Figures 4 and 5 indicate that there is very little variation with $k_{i}^{\prime}$ and $\beta_{i}^{\prime}$ when $k$ is close to 1 and $c a(t)$ and $c^{2} k_{0} W(t)$ decrease with increasing values of $k$. From Fig. 4 we can see that the maximum value of the contact area is achieved at $t=\Delta_{1}, \Delta_{2}$ and is independent of parameters. This latter point is an immediate consequence of equation (3.51) and confirms results given in [6]. Also we note, from these two graphs, that $c a(t)$ and $c^{2} k_{0} W(t)$ achieve their minimum values a little earlier than $c D(t)$ does. This follows from equation (4.6), noting that $\alpha_{i}, \beta_{i}, k_{i}$, and $-l_{i}$, $i=1,2,3, \ldots$, are positive and the denominator of that equation is greater than $l_{e}$, which is positive. Finally the load achieves its maximum a little earlier than contact area and indentation.

(iii) Area-controlled mode. In this case we have

$$
a(t)=M\left(a_{0}-\cos (\omega t)\right), \quad a_{0} \geq 1, \Delta=\frac{2 \pi}{\omega}
$$

and

$$
t_{0}=0, \quad \Delta_{2}=-\Delta_{1}=\frac{\pi}{\omega}, \quad t_{1}(t)=-t .
$$




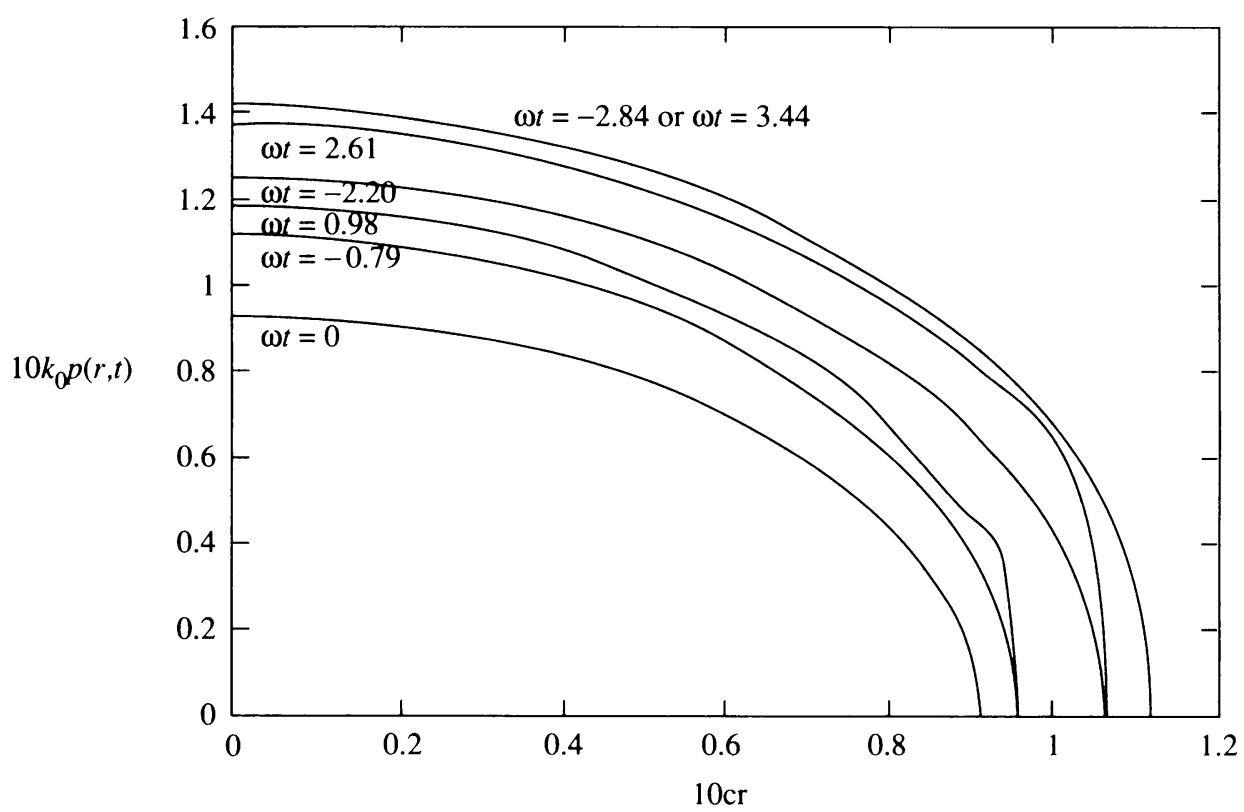

Fig. 3. Pressure distribution for the stress-controlled mode. This picture gives the dimensionless pressure distribution $p(r, t)$ at various times during the cycles for the stress-controlled mode with $k_{1}^{\prime}=0.52$, $k_{2}^{\prime}=0.12, \beta_{1}^{\prime}=0.4, \beta_{2}^{\prime}=0.6, k=2.5$, and $c^{2} K k_{0}=0.0008$. The lines on the top and bottom are for the times when the contact area is maximum and minimum, respectively. The pairs of lines that meet on the horizontal axis are for times when the contact area radii are the same.

Therefore $D(t), W(t)$, and $p(r, t)$ are easier to determine than in the other two cases. From equations $(2.57)-(2.59)$, we can find $D_{e}(t), k_{e} p_{e}(r, t)$, and $k_{e} W_{e}(t)$. Then equations (3.34)-(3.39) are solved numerically using the trapezoidal rule to give $D(t), p(r, t)$, and $W(t)$. Results are presented in Figures 7-9 (see pp. 466, 467). It is clear, from Figure 7 , that the maximum indentation is achieved at $\Delta_{1}, \Delta_{2}$ and is independent of $k_{i}^{\prime}, \beta_{i}^{\prime}$, and $k$ for the area-controlled case. The latter point follows from (3.51). Also we notice, from Figure 8, that total load decreases with increasing $k$ and the minimum value of the total load is achieved at $t=0$ and is dependent only on $k$, the latter observation following from (3.42).

The results on times of maxima and minima of the various quantities in all these modes reflect the general result [6] that load and contact area are simultaneously minimum while indentation and contact area are simultaneously maximum. Also there is the general phenomenon whereby indentation (strain) lags behind load (stress).

From Figures 3, 6, and 9, the pressure distributions for the three modes, we can see the effects of viscoelasticity: the pressures are different even for the same contact area. Note that the tendency of the pressure to develop a hump followed by a sharp decline, that was remarked in $[1,3]$ for positive time $t$, is also demonstrated.

All these results are quite similar to those given by Golden and Graham [3] for the standard linear material, but now we have five parameters $k_{1}, k_{2}, \beta_{1}, \beta_{2}$, and $k_{e}$ (or 


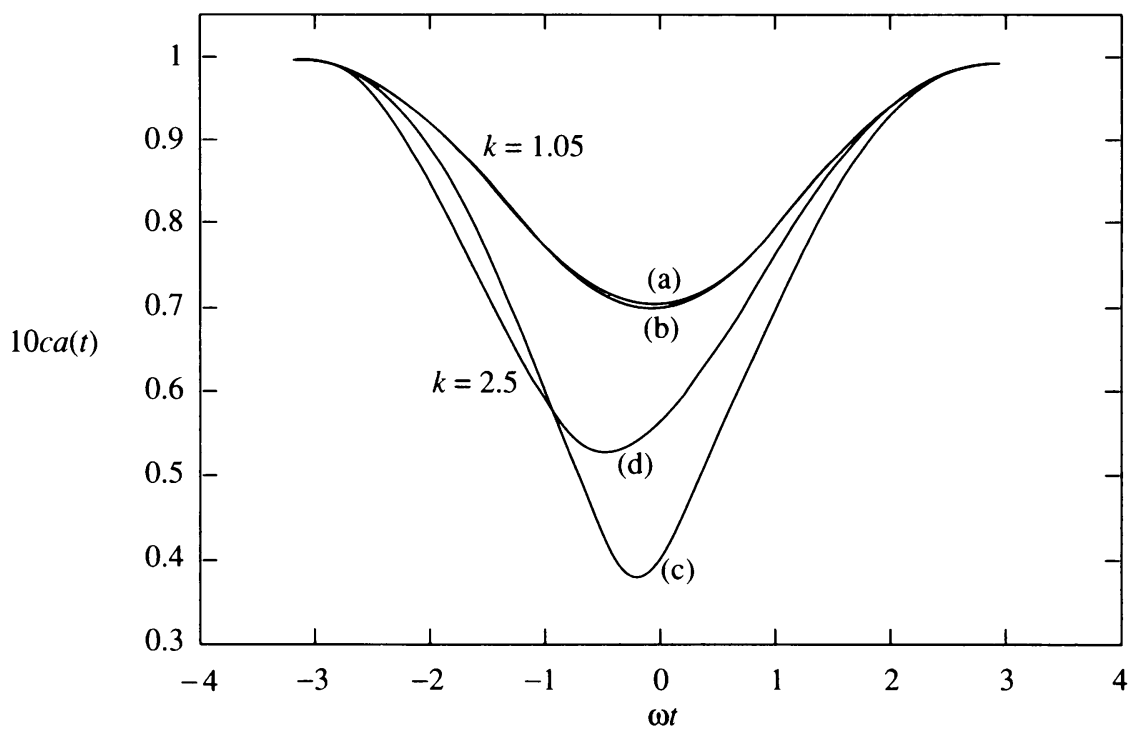

FIG. 4. Contact area radius for strain-controlled mode. This shows the dimensionless contact area radius $c a(t)$ over a complete cycle in the strain-controlled mode for cases (a): $k_{1}^{\prime}=0.0096, k_{2}^{\prime}=0.003$, $\beta_{1}^{\prime}=0.2, \beta_{2}^{\prime}=1.5, k=1.05 ;(\mathrm{b}): k_{1}^{\prime}=0.09, k_{2}^{\prime}=0.03, \beta_{1}^{\prime}=2$, $\beta_{2}^{\prime}=6, k=1.05 ;(\mathrm{c}): k_{1}^{\prime}=1.26, k_{2}^{\prime}=0.12, \beta_{1}^{\prime}=0.9, \beta_{2}^{\prime}=1.2$, $k=2.5$; and (d): $k_{1}^{\prime}=0.52, k_{2}^{\prime}=0.12, \beta_{1}^{\prime}=0.4, \beta_{2}^{\prime}=0.6$, $k=2.5$. Also $c N=0.005$ and $b=3$.

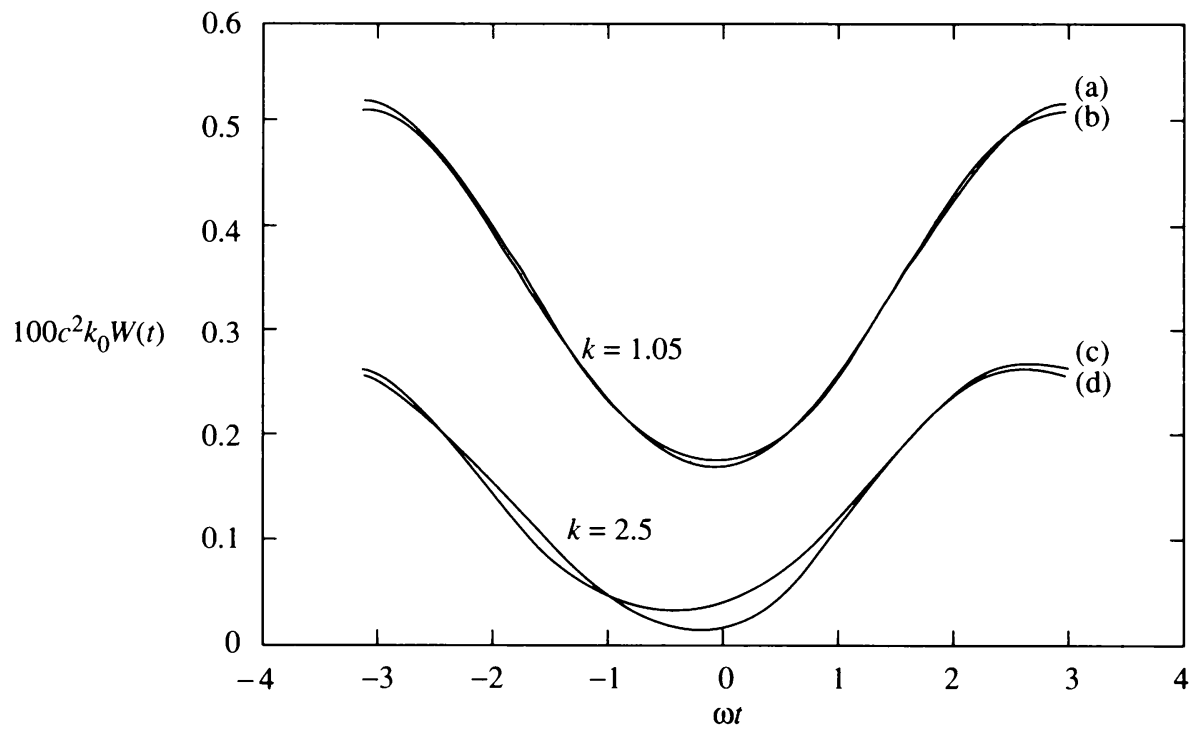

Fig. 5. Total load for strain-controlled mode. It proves the dimensionless total load $c^{2} k_{0} W(t)$ over a complete cycle in the straincontrolled mode for the same four cases as Fig. 4. (a): $k_{1}^{\prime}=0.0096$, $k_{2}^{\prime}=0.003, \beta_{1}^{\prime}=0.2, \beta_{2}^{\prime}=1.5, k=1.05 ;(\mathrm{b}): k_{1}^{\prime}=0.09$, $k_{2}^{\prime}=0.03, \beta_{1}^{\prime}=2, \beta_{2}^{\prime}=6, k=1.05$; (c): $k_{1}^{\prime}=1.26, k_{2}^{\prime}=0.12$, $\beta_{1}^{\prime}=0.9, \beta_{2}^{\prime}=1.2, k=2.5$; and (d): $k_{1}^{\prime}=0.52, k_{2}^{\prime}=0.12$, $\beta_{1}^{\prime}=0.4, \beta_{2}^{\prime}=0.6, k=2.5$. Also $c N=0.005$ and $b=3$. 


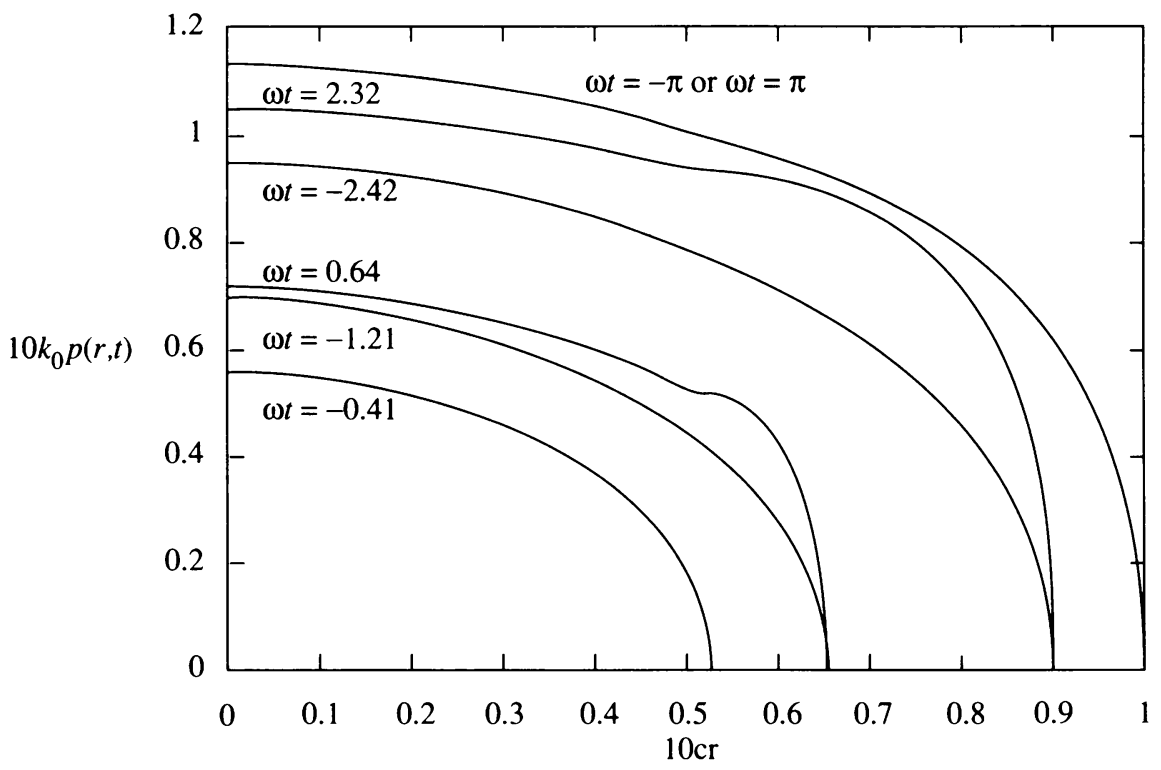

Fig. 6. Pressure distribution for the strain-controlled mode. This gives the dimensionless pressure distribution at different times in a cycle of strain-controlled mode with parameters $k_{1}^{\prime}=0.52, k_{2}^{\prime}=$ $0.12, \beta_{1}^{\prime}=0.4, \beta_{2}^{\prime}=0.6, k=2.5, c N=0.005$, and $b=3$. The lines on the top and bottom are for the times when the contact area is maximum and minimum, respectively. The pairs of lines which meet on the horizontal axis are for times when the contact area radii are equal.

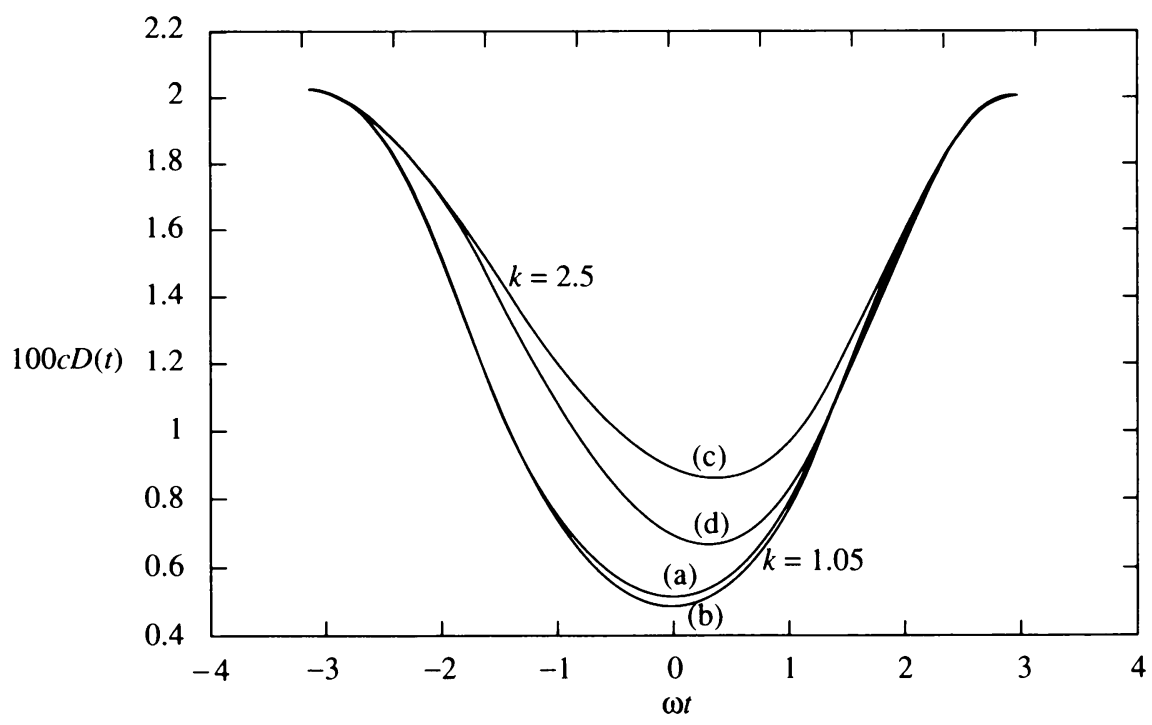

Fig. 7. Indentation for the area-controlled mode. It shows the dimensionless indentation over a complete cycle under the area-controlled condition with parameters $(\mathrm{a}): k_{1}^{\prime}=0.0096, k_{2}^{\prime}=0.003, \beta_{1}^{\prime}=0.2$, $\beta_{2}^{\prime}=1.5, k=1.05 ;(\mathrm{b}): k_{1}^{\prime}=0.09, k_{2}^{\prime}=0.03, \beta_{1}^{\prime}=2, \beta_{2}^{\prime}=6$, $k=1.05$; (c): $k_{1}^{\prime}=1.26, k_{2}^{\prime}=0.12, \beta_{1}^{\prime}=0.9, \quad \beta_{2}^{\prime}=1.2$, $k=2.5$; and (d): $k_{1}^{\prime}=0.52, k_{2}^{\prime}=0.12, \beta_{1}^{\prime}=0.4, \beta_{2}^{\prime}=0.6$, $k=2.5$. Also $c M=0.025$ and $a_{0}=3$. 


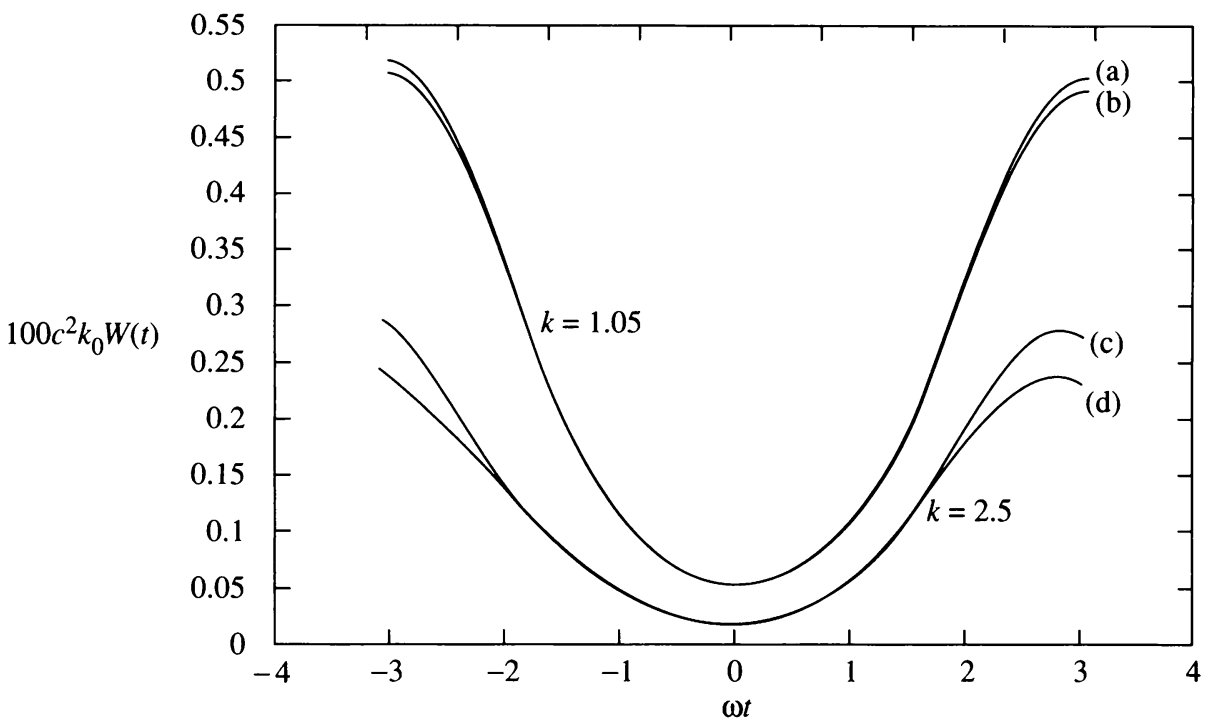

FIG. 8. Total load for the area-controlled mode. This provides the total load over a complete cycle under the area-controlled condition with the same parameters as those for Fig. 7. (a): $k_{1}^{\prime}=0.0096$, $k_{2}^{\prime}=0.003, \beta_{1}^{\prime}=0.2, \beta_{2}^{\prime}=1.5, k=1.05 ;(\mathrm{b}): k_{1}^{\prime}=0.09$, $k_{2}^{\prime}=0.03, \beta_{1}^{\prime}=2, \beta_{2}^{\prime}=6, k=1.05$; (c): $k_{1}^{\prime}=1.26, k_{2}^{\prime}=0.12$, $\beta_{1}^{\prime}=0.9, \beta_{2}^{\prime}=1.2, k=2.5$; and $(\mathrm{d}): k_{1}^{\prime}=0.52, k_{2}^{\prime}=0.12$, $\beta_{1}^{\prime}=0.4, \beta_{2}^{\prime}=0.6, k=2.5$. Also $c M=0.025$ and $a_{0}=3$.

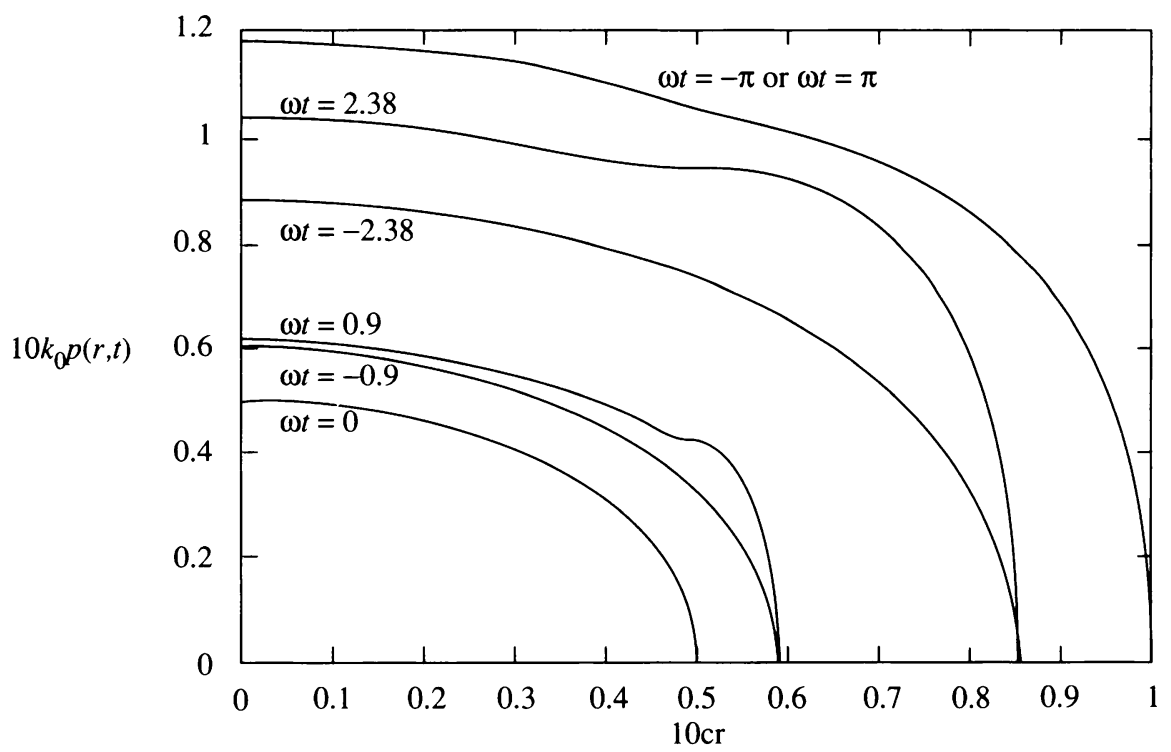

Fig. 9. Pressure distribution of the area-controlled mode. This gives the dimensionless pressure at several times during a period under the area-controlled condition for the case in that $k_{1}^{\prime}=0.52, k_{2}^{\prime}=0.12$, $\beta_{1}^{\prime}=0.4, \beta_{2}^{\prime}=0.6, k=2.5, a_{0}=3$, and $c M=0.025$. The lines on the top and bottom are for the times when the contact area is maximum and minimum, respectively. The pairs of lines that meet on the horizontal axis are for times when the contact area radii are the same. 
$l_{1}, l_{2}, \alpha_{1}, \alpha_{2}$, and $\left.l_{e}\right)$, four of them are independent, instead of three parameters $\alpha, \beta$, and $f$ (two of them are independent) in [3].

Actually, the method used in this paper is applicable to any periodic loading. As we have seen from the above results for the three different loadings, we need to find the initial conditions (e.g., $W\left(t_{0}\right)=W_{e}\left(t_{0}\right)$ for loading; $D\left(\Delta_{1}\right)=D_{e}\left(\Delta_{1}\right)$ for indentation) to begin the numerical calculation. In the general case, formulas for $t_{0}, \Delta_{1}$, and $\Delta_{2}$ can be obtained by using the so-called generalized correspondence principle given in [6].

Acknowledgment. This work was supported by funds provided by the Natural Sciences and Engineering Research Council of Canada. Insights into the behaviour of the solutions reported here were partially provided by numerical explorations done by Mr. Tony Boey, while he was employed on an NSERC Undergraduate Summer Research Award at Simon Fraser University during 1988.

\section{REFERENCES}

[1] J. M. Golden and G. A. C. Graham, The steady-state plane normal viscoelastic contact problem, Internat. J. Engrg. Sci. 25, 277-291 (1987)

[2] G. A. C. Graham and J. M. Golden, The three-dimensional steady-state viscoelastic indentation problem, Internat. J. Engrg. Sci. 26, 121-126 (1988)

[3] J. M. Golden and G. A. C. Graham, Stress, strain and area-controlled modes for the steady-state normal viscoelastic contact problem, Ocean waves mechanics, in Computational fluid dynamics and mathematical modelling (M. Rahman, ed.), Computational Mechanics Publ., Southampton, 1990, pp. 739-753

[4] J. M. Golden and G. A. C. Graham, A fixed length crack in a sinusoidally loaded general viscoelastic medium, in Continuum Mechanics and its Applications (G. A. C. Graham and S. K. Malik, eds.), Hemisphere, Washington, DC, 1989, pp. 171-188

[5] J. M. Golden and G. A. C. Graham, Boundary Value Problems in Linear Viscoelasticity, SpringerVerlag, Berlin, Heidelberg, 1988

[6] G. A. C. Graham and J. M. Golden, The generalized partial correspondence principle in linear viscoelasticity, Quart. Appl. Math. 46, 527-538; 49, 397 (1991) (1988) 